\title{
Interpreting Mucoscopy in Lingual Varicosities and Beyond
}

\author{
Sidharth Sonthalia ${ }^{1}$
}

1 SKINNOCENCE: The Skin Clinic \& Research Centre, Gurungram, India

\begin{abstract}
Key words: dermoscopy, mucoscopy, caviar tongue, lingual varicosities, angiokeratoma
Citation: Sonthalia S. Interpreting mucoscopy in lingual varicosities and beyond. Dermatol Pract Concept. 2019;9(2):146-147. DOI: https://doi.org/10.5826/dpc.0902a11
\end{abstract}

Accepted: January 23, 2019; Published: April 30, 2019

Copyright: $\odot 2019$ Sonthalia. This is an open-access article distributed under the terms of the Creative Commons Attribution License, which permits unrestricted use, distribution, and reproduction in any medium, provided the original author and source are credited.

Funding: None.

Competing interests: The authors have no conflicts of interest to disclose.

Authorship: The author takes responsibility for this publication.

Corresponding author: Sidharth Sonthalia, MD, DNB, MNAMS, SKINNOCENCE: The Skin Clinic \& Research Centre, C-2246, Sushant Lok-1, Block-C, Gurungram, 122009, India. Email: sidharth.sonthalia@gmail.com

\section{Introduction}

The first dermoscopic description of caviar tongue with a beautiful image by Jha et al published in a recent issue of this journal is indeed commendable [1]. However, I wish to highlight few pertinent issues regarding this report and, more important, regarding the interpretation of mucoscopy, perhaps the latest [2] and one of the trickiest realms of dermoscopy.

\section{Comments}

Owing to its noninvasive nature, ease, and promptness of suggesting a diagnosis, for experienced dermatologists dermoscopy is rapidly replacing histopathology for diagnosis of many disorders of general dermatology [3]. However, the above assertion is logically practical only for conditions in which the dermoscopic-histological correlation has been well established, that too in a substantial number of cases, eg, psoriasis, lichen planus, ezcemas, alopecias, and certain skin tumors. Similarly, if the clinical presentation alone may be sufficient for a correct diagnosis (as in many mucous membrane conditions including caviar tongue), then researchers may of course comment on their dermoscopic appearance but prefer not to label the latter as diagnostic hallmarks. Also, when the dermoscopic features of a skin or mucosal condition are being documented for the first time in scientific literature, its validation against the gold standard diagnostic criteria becomes essential. While the diagnosis of caviar tongue is nearly always clinical, differentials including hemangioma, lymphangioma, Kaposi sarcoma, and mucosal melanoma are worth consideration. Notwithstanding the clinical certainty of the case described by Jha et al being caviar tongue, in the absence of confirmatory histopathology, the dermoscopic features described should not be unduly glorified as an "auxiliary tool for its diagnosis" lest there is sufficient evidence (not a single patient's findings) and correlation with histopathology (the current gold standard of diagnostic confirmation) has been established.

I also wish to highlight that the mutual contradiction between the following authors' statements should be avoided in future such reports: "To the best of our knowledge, this is the first report on dermoscopy of caviar tongue" and " $\ldots$ based on location, age, clinical and dermoscopic appearance, it was diagnosed as lingual varicosities." If as per the authors' own assertion this was the first published report on 
dermoscopy of caviar tongue, then how could it simultaneously be taken as one of the criteria for diagnosis, that too in the absence of histological confirmation?

Authors should be more careful about the use of terminology for dermoscopic characterization. Pending the algorithm and scheme of systematic description of mucoscopic findings, I believe and suggest that mucoscopic description should include at least certain minimum parameters (Table 1).

In my opinion, the complete description of the dermoscopic image of caviar tongue displayed in the concerned report should have been as follows: absence of pigmentation, presence of multiple loosely scattered red lacunae with bluish white (rather than white) veil present over a yellowish crimson background, surrounded and interspersed with fine arborizing vessels and scattered telangiectasias and focal white structureless areas (Figure 1).

While the authors dwelled upon the dermoscopic differentiation from hemangioma and lymphangioma, it would have been better to mention the lack of melanocytic structures and rainbow pattern for formal differentiation from mucosal melanoma and mucosal Kaposi sarcoma, respectively, which constitute closer clinical differentials of caviar tongue than pyogenic granuloma. And although oral angiokeratomas are relatively rare and typically involve the tip or the dorsum of the tongue [4], mentioning them in the discussion may have been better than discussing angiokeratomas of Mibelli.

\section{Conclusions}

Dermoscopy is not a new technology, but its exploration for noninvasive diagnosis in general dermatology, including mucosal disorders, is a relatively recent phenomenon. While acquiring the skills to use this technique is no longer "optional" for dermatologists, great caution must be exercised while capturing the image and interpreting and reporting features according to the best and latest available guidelines. Mucoscopy warrants extra care in this regard. Thus I request all dermatology colleagues who are practicing and reporting dermoscopic findings of different dermatoses to descry the science by analysis, not nonchalance, and master the art with patience, not haste [5].

\section{References}

1. Jha AK, Zeeshan MD, Jha Amar AK. Mucoscopy in lingual varicosities. Dermatol Pract Concept. 2018;8(1):54-55.

2. Sonthalia S, Varma S, Jha AK, Jakhar D, Kaliyadan F. Case report: dermoscopic features of oral lichen planus-the evolution of mucoscopy. Version 2. F1000Res. 2018 Mar 6 [revised 2018 Mar 27];7:284.

3. Sonthalia S, Errichetti E. Dermoscopy—not just for diagnosis and not just for dermatologists! Kathmandu Univ Med J (KUMJ). 2017;15(57):1-2.
Table 1. Suggested Minimum Parameters for Adequate Dermoscopic (Mucoscopic) Characterization of a Mucosal Lesion

\begin{tabular}{|l|l|}
\hline Parameter/Structure & \multicolumn{1}{|c|}{ Characteristics } \\
\hline Melanocytic criteria & Absent/present/suspicious ${ }^{\mathrm{a}}$ \\
\hline Veil & $\begin{array}{l}\text { Present/absent } \\
\text { Color: white, bluish white }\end{array}$ \\
\hline Background & $\begin{array}{l}\text { Color } \\
\text { Homogeneity }\end{array}$ \\
\hline including lacunae & $\begin{array}{l}\text { Absent/present } \\
\text { Distribution } \\
\text { Morphology } \\
\text { For lacunae (lagoons) } \\
\text { Variant (red and/or dark) } \\
\text { Distribution within the lesion }\end{array}$ \\
\hline $\begin{array}{l}\text { Additional/specific } \\
\text { dermoscopic feature(s) } \\
\text { (if present) }\end{array}$ & $\begin{array}{l}\text { For example } \\
\text { Structureless areas } \\
\end{array}$ \\
\hline
\end{tabular}

a Since mucous membrane architecture is different from that of the skin and conventional melanocytic criteria such as brown globules or lines are frequent in nonmelanocytic lesions, this terminology of "melanocytic criteria-absent/present/suspicious" may be replaced with a more descriptive approach, eg, presence of a particular pattern of pigmentary network.

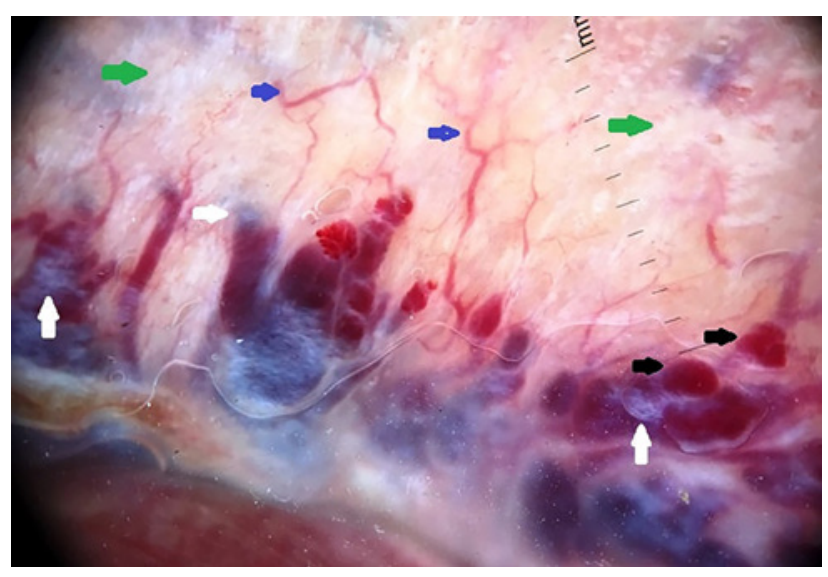

Figure 1. Reannotated Figure 2 of the original case report [1]. The mucoscopic image shows a yellowish crimson background with multiple loosely scattered red lacunae (black arrows) with bluish white veil (white arrows), interspersed fine arborizing vessels (blue arrows), and scattered telangiectasias and focal white structureless areas (green arrows) (polarized dermoscopic image from the original case, $\times 10)$. [Copyright: $@ 2018$ Jha et al]

4. Ranjan N, Mahajan VK. Oral angiokeratomas: proposed clinical classification. Int J Dermatol. 2009;48(7):778-781.

5. Sonthalia S, Jha AK, Bosseila M, Errichetti E. Dermoscopy-master by analysis and patience, not haste and nonchalance. Pigment Int. 2018;5:117-119. Available from: http://www.pigmentinter national.com/text.asp?2018/5/2/117/247509. Accessed on January 23, 2019. 